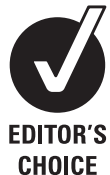

CHOICE
${ }^{1}$ Department of Cellular Pathology, John Radcliffe Hospital/University of Oxford, Oxford, UK

${ }^{2}$ Nuffield Department of Clinical Laboratory Sciences, Biomedical Research Centre, John Radcliffe Hospital/University of Oxford, Oxford, UK

\section{Correspondence to} Professor Runjan Chetty, Department of Cellular Pathology, Level 1, Academic Centre, John Radcliffe Hospital, Headley Way, Oxford, OX3 9DU, UK

runjan.chetty@ndcls.ox.ac.uk

Accepted 18 April 2011 Published Online First 18 May 2011

\title{
Follicular patterned lesions of the thyroid gland: a practical algorithmic approach
}

\author{
Runjan Chetty ${ }^{1,2}$
}

\section{ABSTRACT}

Follicular patterned lesions of the thyroid are problematic and interpretation is often subjective. While thyroid experts are comfortable with their own criteria and thresholds, those encountering these lesions sporadically have a degree of uncertainty with a proportion of cases. The purpose of this review is to highlight the importance of proper diligent sampling of an encapsulated thyroid lesion (in totality in many cases), examination for capsular and vascular invasion, and finally the assessment of nuclear changes that are pathognomonic of papillary thyroid carcinoma (PTC). Based on these established criteria, an algorithmic approach is suggested using known, accepted terminology. The importance of unequivocal, clear-cut nuclear features of PTC as opposed to inconclusive features is stressed. If the nuclear features in an encapsulated, non-invasive follicular patterned lesion fall short of those encountered in classical PTC, but nonetheless are still worrying or concerning, the term 'uncertain malignant potential or behaviour, most likely benign' is suggested. Indubitable, classical PTC nuclei (whether diffuse or restricted to a single high-power field) are diagnostic of a PTC be it classical, non-invasive or invasive follicular variant PTC. Capsular and vascular invasion remain the only reliable predictors of outcome, as non-invasive, encapsulated follicular variant PTC, even with diffuse PTC nuclear change, behaves in an indolent fashion.

\footnotetext{
"The small part of ignorance that we arrange and classify, we give the name of knowledge." Ambrose Bierce.
}

\section{INTRODUCTION}

Classical pathology has taught us that cancer is defined and typified by its invasive growth characteristics. Invasion, in turn, is characterised by the tumour spreading beyond its immediate confines (basement membrane, or pertinent to the thyroid, the capsule) and then into vessels or via natural passages. With few exceptions, tumours that have metastasised are assumed, de facto, to be malignant. These fundamental pathology mantras are applied on a daily basis in distinguishing benign from malignant. However, the thyroid gland represents a different set of circumstances for the assessment of invasion and thus cancer. Basement membrane invasion and stromal desmoplasia are not relevant. When confronted by a follicular patterned tumour, the pathologist relies on capsular and/or vascular invasion to determine the biological behaviour of a thyroid tumour. As in many areas of pathology, the issue of subjectivity has plagued the interpretation of what superficially appears to be mundane: what is 'true' capsular and vascular invasion? While strenuous attempts have been made to qualify exactly what constitutes capsular and vascular invasion, somewhat anecdotal interpretations still exist. The next and still current, controversy concerning thyroid pathologists is the interpretation of 'papillary thyroid cancer nuclear features' and what they mean in the context of an encapsulated, non-invasive follicular patterned lesion. Several pathologists have attempted to address this and related issues on classification with varying success and inconstant application. The rate limiting steps have been the absence of reproducible morphological, immunohistochemical and molecular markers that enable pathologists to stratify the contentious thyroid neoplasms reliably according to biological behaviour. This has been compounded by the absence of large studies with long-term follow-up until fairly recently. Characteristic molecular signatures of the different lesions have not emerged, but molecular patterns are beginning to take shape. Hence, any classification and nomenclature that may be advanced currently, is in evolution, at best.

\section{HISTORICAL ASPECTS}

Before looking forward, it is worth dwelling on some of the key historical aspects that have led us to the current state of play. At one time, encapsulated follicular lesions of the thyroid were simple: if there was no capsular or vascular invasion, it was a thyroid follicular adenoma. If there was, then it was a follicular carcinoma.

The description of a follicular neoplasm containing cells bearing the characteristic nuclear features of a papillary thyroid cancer, led to the introduction of the term, 'follicular variant of papillary carcinoma', into the thyroid pathology lexicon. ${ }^{1}{ }^{2}$ When such tumours showed capsular and/or vascular invasion, reporting pathologists felt comfortable using the appellation 'carcinoma', and treating physicians felt comfortable in proceeding with the appropriate therapeutic regimen based on that diagnosis.

Encapsulated papillary patterned neoplasms harbouring the classical nuclear features, did not present a diagnostic problem as the papillary architecture and nuclear features were sufficient diagnostic hooks on which a confident diagnosis could be rendered, irrespective of whether capsular and/or vascular invasion was present. ${ }^{34}$

Encapsulated follicular variant of papillary thyroid carcinoma (EFVPTC) was regarded in a similar light to encapsulated papillary carcinoma in terms of biological behaviour. In the presence of invasion (capsular and/or vascular), these lesions presented 
little or no debate. The EFVPTC with invasion was in essence the same as the papillary equivalent but instead of papillary structures, the lesion was composed of follicular structures. 56

Fundamentally, if one agrees that the cell of origin of thyroid neoplasms is the thyroid follicle-lining cell, then a neoplasm arising from these cells may have either papillary or follicular growth patterns, and they may share similar nuclear features/ changes. It is perhaps a historical quirk that a papillary neoplasm containing characteristic nuclear features was described and recognised before a similar follicular patterned neoplasm. But for this, we would be talking about a 'papillary variant of a follicular neoplasm'! Indeed, this is precisely what Lindsay ${ }^{1}$ regarded these tumours as (Juan Rosai, personal communication, 2011).

The contentious issues at the moment are the interpretation of follicular neoplasms that are encapsulated, non-invasive and show focal/'incomplete' or widespread/diffuse nuclear changes that are diagnostic of papillary thyroid cancer. How should these lesions be diagnosed, classified and, most importantly, treated?

Most, if not all, pathologists are comfortable calling a neoplasm with papillary architecture and characteristic nuclear changes, a carcinoma irrespective of invasion. Why shouldn't the same principles be applied to a follicular patterned neoplasm lacking invasion but showing similar nuclear features? The crux lies in the degree and extent of the nuclear changes. Most would agree that a follicular-patterned lesion showing 'full house', diffusely present, unequivocal nuclear features of papillary thyroid carcinoma (PTC), should be diagnosed as a follicular variant of papillary carcinoma, irrespective of the presence or absence of invasion. Invasion is not a diagnostic essential, as up to $27 \%$ of non-invasive encapsulated papillary carcinomas can present with lymph node involvement. ${ }^{7}$ Hence, a precedent exists for a non-invasive thyroid neoplasm having the capability and potential of metastasising. The mechanism by which encapsulated, non-invasive thyroid neoplasms (either papillary or follicular) spread to lymph nodes is possibly via intra-thyroid lymphatics or other related mechanism.

This leaves the vexing residue of non-invasive follicular patterned neoplasms with partial or incomplete nuclear features of PTC. What this encompasses varies from observer to observer and ranges from a few cells showing nuclear grooves to a cluster of cells showing some, but not all the nuclear features typically associated with PTC. Some pathologists are happy with minimal criteria (one or two cells showing nuclear grooves only) to render a diagnosis of EFVPTC and have the patient treated as for classical PTC. Others feel more reluctant to make the diagnosis and have the attendant cancer treatment inflicted on the patient. Several have looked at this problem, highlighting the diagnostic variability among experts, ${ }^{8}$ and others have suggested stringent criteria for the diagnosis of FVPTC. ${ }^{9}$ The Chernobyl Pathologist Group suggested a compromise appellation for the cohort of tumours showing incomplete or focal PTC nuclear changes. ${ }^{10}$ The term 'uncertain malignant potential' was born and is in use in other organs such as the bladder. While this is a convenient term for pathologists, the clinical and biological ramifications of this term have caused concern among clinicians. Rather than guiding their therapeutic options, they were uncertain how to treat these 'uncertain malignant potential' lesions. Despite this, the term is being used more frequently among pathologists and may well have a role as a legitimate diagnostic category.

\section{RECENT CLINICOPATHOLOGICAL AND MOLECULAR STUDIES}

The question one has to address is how does our interpretation of a constellation of histological features in the absence of invasion actually translate into the behaviour, management and prognosis of this subset of thyroid tumours? Ancillary aids such as immunohistochemistry for CK19, Galectin-3 and HBME-1 and, indeed, the molecular profiles, have not resulted in a reliable, reproducible ability to stratify these lesions that correlates with behaviour. What molecular analyses do indicate currently is that the EFVPTC harbours RAS mutations rather than $B R A F$ mutations, which is more in keeping with the molecular profile of follicular adenoma and carcinoma. ${ }^{11}$ Once these lesions show infiltrative characteristics, $B R A F$ mutations then dominate and the tumour has the same profile as classical PTC. ${ }^{11} B R A F$ mutations only occurred in FVPTC and this was not the V600E mutation that is encountered in classical PTC. ${ }^{11}$ In another study by Castro and colleagues, PAX8/PPAR $\gamma$ and RAS mutation rates were found to be at the same level in FVPTC, follicular adenoma and follicular carcinoma. ${ }^{12}$

Despite these advances, morphologic examination and interpretation remains the gold standard and only means of categorisation in many cases.

Key to the examination of encapsulated thyroid lesions is capsular and/or vascular invasion and their extent. The weight of the current literature indicates that vascular invasion has more sinister connotations for biological behaviour than capsular invasion.

The study by Goldstein and colleagues compared metastatic and non-metastatic minimally invasive follicular carcinoma. ${ }^{13}$ This study indicates that metastatic potential is related to vascular invasion rather than capsular penetration. Both nonmetastatic and metastatic lesions had the same number of complete capsular penetrations. ${ }^{13}$ However, all encapsulated follicular lesions that metastasised, demonstrated vascular invasion. ${ }^{13}$ This study indicates that as long as the capsular invasion does not extend into surrounding thyroid tissue and beyond, then 1-3 foci of capsular penetration, is associated with a good prognosis. This begs the question: how important is capsular invasion that just goes through the capsule? If left untreated, would these then proceed to extend into non-tumour thyroid tissue and beyond? This obviously is a matter of conjecture. It is difficult to ignore a basic tenet of aggressive tumour behaviour (capsular invasion), even if long-term followup does not show recurrences or relapses. Tradition would decree that an encapsulated follicular lesion lacking PTC nuclei but showing even 1 focus of complete capsular penetration, should be flagged. It is likely that such a tumour be placed in the "most likely benign' or uncertain malignant potential category.

The next issue that needs to be addressed is the significance of PTC nuclei in an encapsulated follicular neoplasm showing none or even up to $1-3$ foci of capsular penetration, but no vascular invasion (after complete sampling of the capsule of the lesion). As Dr Rosai stated eloquently, there is much nomenclatural conjecture in this area of thyroid pathology. ${ }^{14}$ To classify a lesion as a carcinoma without two of the classical attributes of a cancer (capsular and vascular invasion) is difficult to reconcile with traditional thinking. Such examples have been reported to present with metastasis in spite of the absence of capsular and/ or vascular invasion. ${ }^{15}$ The obvious response and counter is that invasion (especially vascular) may have been missed in these cases even though due diligence was exercised and the entire lesion was sampled and processed. Such cases are fortunately extremely rare. Recent detailed clinicopathological studies with long-term follow-up ( $>10$ years) indicate that non-invasive FVPTC behave well and that lobectomy alone is sufficient treatment. ${ }^{16}$ In other words, they behave like follicular adenomas and this is supported by shared molecular changes in both lesions. But it is extremely difficult to label a lesion, 
showing even 1 focus of true capsular invasion, a banal follicular adenoma, irrespective of long-term follow-up evidence.

To say that PTC nuclear features alone confer the appellation of FVPTC on encapsulated, non-invasive lesions is at odds with the molecular evidence that points towards similarity with follicular adenoma. ${ }^{11}$ Given that FVPTC is an accepted diagnostic entity, it then implies it is the nuclear features rather than the architecture that defines the entity, which again is at odds with traditional nomenclatural convention. This is where the molecular evidence accrued so far can perhaps shed some light. If a thyroid follicle-lining cell acquires a KRAS mutation, then the subsequent neoplasm tends to have a follicular growth pattern: if there is a BRAF (V600E) mutation, then the growth pattern tends to be papillary. It is likely that other and/or further molecular derangements are required before the morphologically recognisable tumour stage is reached. When and how the characteristic nuclear changes arise is not known. Furthermore, the current state of our molecular knowledge does not shed any light on it because FVPTC does not share the same genetic aberrations as classical PTC, with or without invasion.

\section{INTERPRETATION OF NUCLEAR CHANGES ASSOCIATED WITH PTC}

Anyone doing thyroid pathology today is obsessed with looking for the characteristic nuclear changes that are associated with PTC in almost every thyroid specimen. The set of nuclear features that define PTC have been seen focally in a variety of tumour and non-tumour conditions in the thyroid and obviously context, not to mention common sense, ought to prevail in the interpretation of such changes. When the PTC nuclear changes are unequivocal, florid, pervasive and in 'full flower', then encapsulated, follicular patterned lesions (with or without invasion) should be labelled as FVPTC. ${ }^{14}$ This is the prevailing wisdom despite the molecular caveats that are mentioned above. Having said that, the vast majority of these tumours behave in an indolent fashion, but if one uses the appellation PTC, then they should be treated as such.

If the nuclear changes of PTC are only focal and/or incomplete then it is a matter of personal preference how these lesions are labelled. Some may call them FVPTC based on a few cells showing longitudinal nuclear grooves, other may require more of the typical cytological changes to be present. The lack of consensus of what represents minimum criteria both in terms of quantity (what is focal?) and quality (how many PTC nuclear criteria are required?) remains. ${ }^{8}$ If such lesions show capsular invasion through the capsule and into surrounding thyroid parenchyma without vascular invasion, it is clearly prudent to regard such invasive lesions as FVPTC, irrespective of the extent of PTC nuclear change. It is in this area that subjectivity reigns and rigid quantification is not possible. Clinicopathological studies indicate benign outcomes for this category of follicular thyroid neoplasm even after treatment by lobectomy alone. There is a school of thought that advocates labelling lesions with partial/incomplete PTC nuclear changes as FVPTC and thus giving the benefit of the doubt to the patient who should be treated as fully-fledged FVPTC. It is axiomatic that if an indolent lesion is treated aggressively it is unlikely to recur or metastasise. Herein lies the dilemma: over-treat and label a patient as having a carcinoma, or not label the lesion carcinoma and advocate lobectomy alone knowing that such lesions do behave indolently over at least a 10-year follow-up period. It is unlikely that adherents to either viewpoint will change their views. However, the greater body of evidence rather than anecdote should dictate clinical practice.
The term 'uncertain malignant potential' has not taken root among clinicians yet (perhaps partly because the term suggests that we as pathologists are 'uncertain' of what we are diagnosing) because they need a clear indication of the category and behaviour of a lesion so that they can institute appropriate therapy. However, with its increasing use, it is likely that more clinicians will become familiar with the group of tumours that are classified as such, leading to more clear-cut treatment options. If 'uncertain malignant potential' is not an acceptable term, then an alternative approach to these lesions is suggested. It is a matter of finding terminology and criteria that are accurate, reproducible and clinically meaningful.

There is an element of angst and uncertainty among nonexpert pathologists who do encounter follicular patterned lesions every so often and there is a need for uniform guidelines. It is worth remembering that non-invasive encapsulated follicular patterned tumours, irrespective of nuclear and indeed, cytoplasmic (oncocytic or non-oncocytic) features, behave in an indolent fashion even after non-cancer treatment protocols. It is imperative that careful examination and processing of the specimen is adhered to as submission of the capsule in toto is desirable and even mandatory. Due diligence should be exercised to exclude the presence of capsular and/or vascular invasion as these remain the basic and most reliable and reproducible tenets of biological behaviour. The type of capsular invasion should be assessed (whether into surrounding thyroid tissue or not) and interpreted in conjunction with vascular invasion (if any) and the nuclear cytological features.

The biggest issue in any suggested approach to follicular patterned tumours, is how best to address the question of subjectivity surrounding the interpretation of the PTC nuclear features in non-invasive tumours. This centres on which and how many of the constellation of nuclear features associated with PTC are required for the diagnosis. Elsheikh et al listed the important nuclear features that the panel of experts ranked as their criteria to make a diagnosis of PTC. ${ }^{8}$ Even in classical PTC, every case shows variability in the nuclear features. In other words, some cases may lack or have occasional intra-nuclear pseudo-inclusions but have all other features. No one feature diagnostically outweighs or is more important than another. It may be sensible to suggest that the observer is in no doubt that the nuclear changes observed fulfil criteria that make the nuclei clearly different from those in non-lesional thyroid tissue or non-neoplastic follicles. At the end of the day, the individual pathologist should feel comfortable to make a diagnosis of FVPTC on unequivocal, pathognomonic features.

The next quandary is quantification: how many cells should contain the typical PTC nuclear features to constitute a diagnostic focus? I think most pathologists would hesitate to diagnose any cancer let alone FVPTC based on one cell showing the

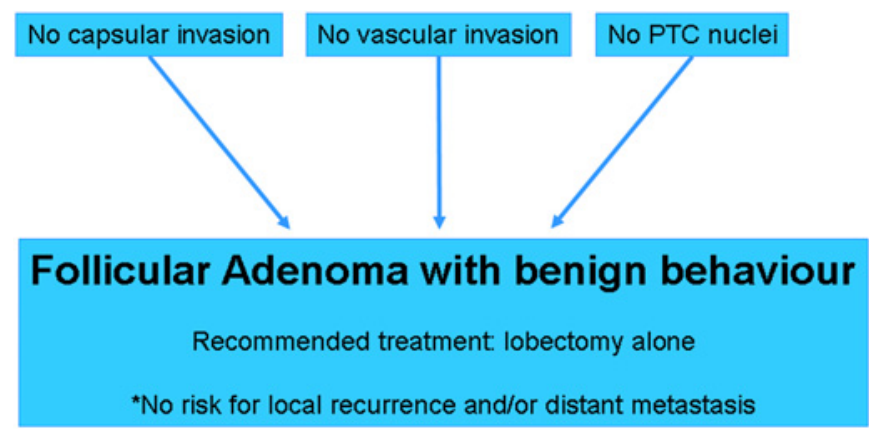

Figure 1 Follicular adenoma with benign behaviour. 


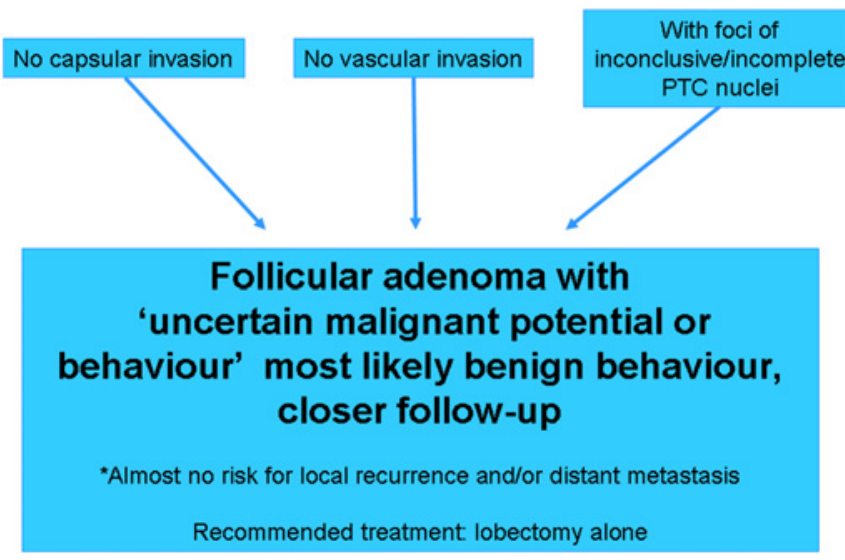

Figure 2 Follicular adenoma with uncertain malignant potential or behaviour.

typical nuclear features. If cancer is due to a field affect and evolves from a clone of tumour cells, then perhaps all the cells within at least one high-power field showing fully-fledged, unequivocal PTC nuclei could be accepted as a minimum criterion. Anything less, that is, so called 'incomplete' or equivocal nuclear change, should not be regarded as diagnostic. The nuclear features of PTC are recognisable by all when obvious and clear-cut. If this gestalt reaction does not occur and one debates whether the occasional nucleus possible could have some features but they are unconvincing and not clear-cut, then they are in all likelihood, not PTC nuclei. The gold standard should be a comparison to the nuclear changes seen in a classical PTC, and re-examining an example of this when pondering whether a follicular patterned lesion contains PTC nuclei or not, certainly does help re-calibrate the eye. If the nuclear changes are inconclusive or 'incomplete' (and there are some cases that resist falling into line neatly!), then the best repository for such lesions is the category of 'uncertain malignant potential/biological behaviour'. Since these lesions have been associated with good outcome (neither metastasising nor recurring), I suggest that the accompanying comment, "most likely benign behaving with almost no risk for recurrence or metastasis" to reflect the published clinical outcomes documented thus far. It may serve as an aid to the oncologists planning the management of these cases.

Next, what constitutes multifocality? Should a particular percentage of tumour cells show PTC nuclear change before crossing the line from follicular adenoma to FVPTC? As far as I am aware there are no published long-term follow-up studies expressly looking at extent (number of foci or percentage) of PTC nuclei in follicular patterned tumours that have been diagnosed as follicular adenomas or FVPTC. Applying the theory

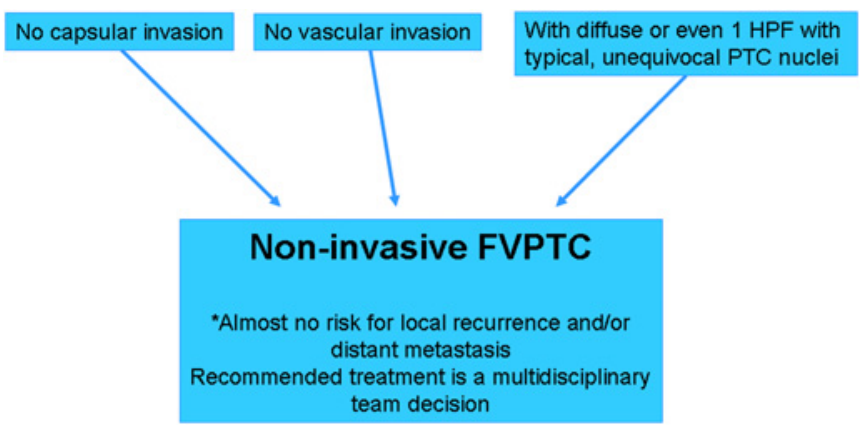

Figure 3 Non-invasive follicular variant papillary thyroid cancer.

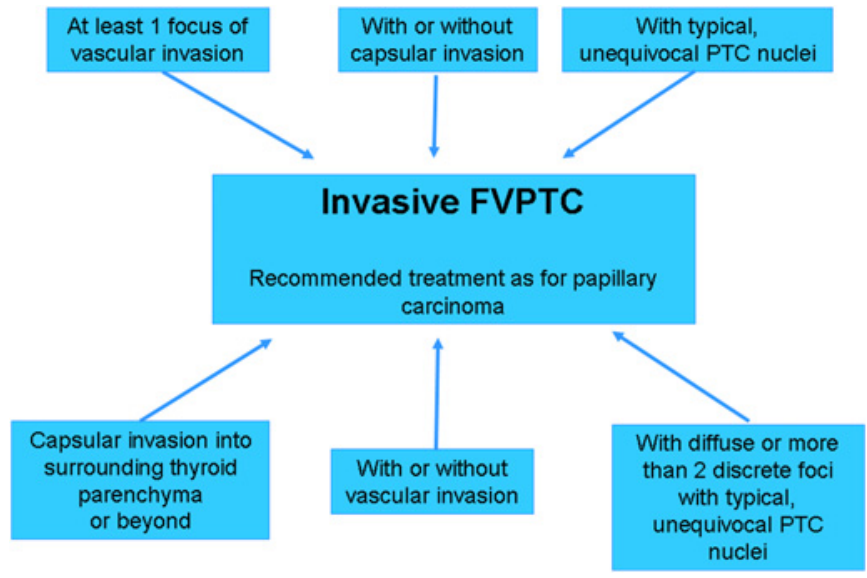

Figure 4 Invasive follicular variant papillary thyroid cancer.

of Occam's razor, perhaps only one high-power field of typical, diagnostic, clear-cut unequivocal PTC nuclei is enough to qualify a lesion as a FVPTC. Given the importance attached to PTC nuclear features, even a single high-power field containing nuclei with unequivocal features in a tumour lacking capsular and vascular invasion should raise concern and merit a diagnosis of non-invasive FVPTC. Molecular analyses of single foci/nodules of cells with PTC nuclear change are a snapshot of a temporal sequence of events and not necessarily a true reflection of the full spectrum of biological behaviour of that particular focus. It depends when in the biological life of the lesion the molecular analysis was performed. An important caveat worth remembering is that temporal molecular events in the genesis of these tumours are not known. The unifying morphological feature between classical PTC and FVPTC is the typical nuclear change. Gene profiling has shown that classical PTC and FVPTC share gene expression profiles that are distinct and separate from follicular adenomas. ${ }^{17}$ Given the strong hierarchical clustering demonstrated in this study ${ }^{17}$ and the shared morphology, there is little doubt that classical PTC and FVPTC are morphological endpoints of the same disease: one with a papillary pattern and the other with a follicular pattern. However, such clear molecular evidence is not apparent when analysing genes that are commonly implicated in thyroid carcinogenesis. If unequivocal PTC nuclear features are diffusely or omnipresent in a noninvasive lesion, this should be diagnosed as non-invasive FVPTC At the other end of the spectrum, if only one or even two high power fields contain unequivocal PTC nuclei and the rest of the

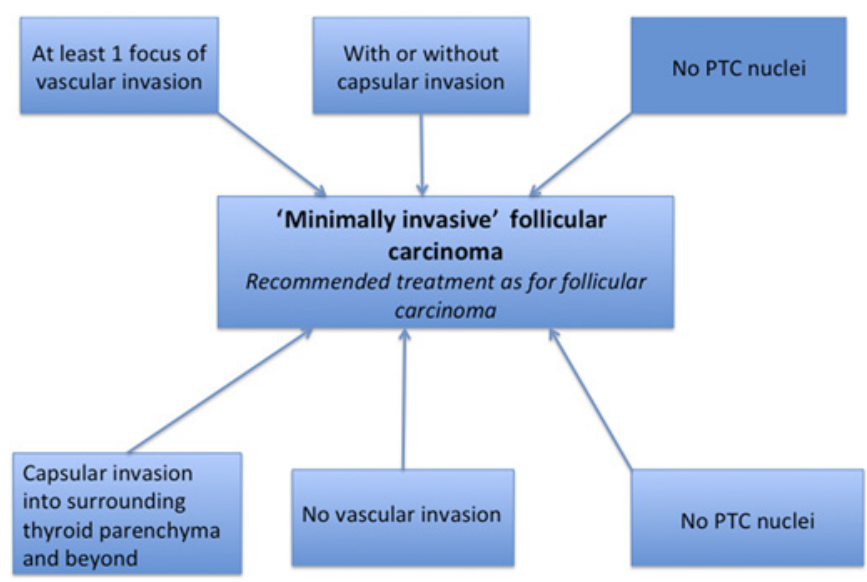

Figure 5 Minimally invasive follicular carcinoma. 


\section{Take-home messages}

Proper handling and sampling of the specimen is advocated.

- If possible the entire capsule should be routinely sampled.

- Nuclear features of PTC should be convincing, clear-cut, readily apparent and not equivocal.

- Even as little as 1 HPF of unequivocal clear-cut PTC nuclei should be considered diagnostic of FVPTC.

- All the nuclear features of PTC may not be present.

- If unequivocal, clear-cut PTC nuclear features are present focally in a nodule, the entire nodule should be regarded as FVPTC.

lesion contains non-PTC nuclei, given the diagnostic importance of PTC, the lesion by definition should also be labelled noninvasive FVPTC. The therapeutic options are very much dependent on a multidisciplinary team approach taking into account all clinicopathological parameters including the individual patient characteristics, imaging and local institutional guidelines, and bearing in mind that non-invasive FVPTC behaves indolently.

\section{CONCLUSIONS}

It is generally accepted that PTC nuclear change is diagnostic of a particular type of thyroid malignancy and if unequivocally present, irrespective of extent, it would be remiss of the pathologist not to document its presence and diagnose the lesion as a PTC. Given the biological uncertainties, the vagaries and unpredictability of individual tumours and patients, perhaps it is best to exclude 'multifocality' as a diagnostic necessity.

However, it does appear that the behaviour of follicular patterned neoplasms with typical, unequivocal PTC nuclear change seems to be dependent on the extent of these changes: tumours lacking multifocal PTC nuclear change and no invasion, behave indolently.

The purpose of this overview is to highlight the difficulties that a practising, non-expert pathologist (such as the author of this piece) encounters whenever confronted by a follicular patterned neoplasm. The crux of this article is to take what has been published in the literature in terms of outcome of follicular patterned lesions of all designations, and attempt to construct a practical algorithmic approach (see figures $1-5$ ) based on the aforementioned literature. While the suggestion is 'theoretical' and not meant to be a new classification system but rather a diagnostic algorithm, it borrows heavily from the published literature (especially clinicopathological series with large numbers of cases with long-term follow-up, and published molecular evidence thus far) and pre-existing nomenclature in trying to provide practising pathologists with practical pointers.

It must be stressed that the author is neither attempting to reinvent the wheel nor trying to obfuscate the real life practical difficulties associated with follicular patterned thyroid neoplasms. While this approach may be construed as theoretical or unduly simplistic with a liberal amount of assumption (such as removing multifocality as a diagnostic consideration), the essential pathological criteria on which it is based are reproducible and time-honoured, and importantly, have pathological and biological significance (especially vascular invasion).

Competing interests None declared.

Provenance and peer review Not commissioned; externally peer reviewed.

\section{REFERENCES}

1. Lindsay S. Carcinoma of the Thyroid Gland:a Clinical and Pathologic Study of 293 Patients at the University of California Hospital. Springfield, Illinois: Charles C Thomas 1960:40-52.

2. Chen KT, Rosai J. Follicular variant of thyroid papillary carcinoma: a clinicopathologic study of six cases. Am J Surg Pathol 1977;1:123-30.

3. Schröder S, Böcker W, Dralle H, et al. The encapsulated papillary carcinoma of the thyroid. A morphologic subtype of the papillary thyroid carcinoma. Cancer 1984; 54:90-3.

4. Evans HL. Encapsulated papillary neoplasms of the thyroid. A study of 14 cases followed for a minimum of 10 years. Am J Surg Pathol 1987;11:592-7.

5. Ghossein R. Encapsulated malignant follicular cell-derived thyroid tumors. Endocr Pathol 2010;21:212-18.

6. Baloch ZW, Shafique K, Flannagan M, et al. Encapsulated classic and follicular variants of papillary thyroid carcinoma: comparative clinicopathologic study. Endocr Pathol 2010;16:952-9.

7. Rivera M, Tuttle RM, Patel S, et al. Encapsulated papillary thyroid carcinoma: a clinico-pathologic study of 106 cases with emphasis on its morphologic subtypes (histologic growth pattern). Thyroid 2009;19:119-27.

8. Elsheikh TM, Asa SL, Chan JK, et al. Interobserver and intraobserver variation among experts in the diagnosis of thyroid follicular lesions with borderline nuclear features of papillary carcinoma. Am J Clin Pathol 2008;130:736-44.

9. Chan JKC. Strict criteria should be applied in the diagnosis of encapsulated follicular variant of papillary thyroid carcinoma. Am J Clin Pathol 2002;117:16-18.

10. Williams ED. Guest editorial: two proposals regarding the terminology of thyroid tumors. Int J Surg Pathol 2000;8:181-3.

11. Rivera M, Ricarte-Filho J, Knauf J, et al. Molecular genotyping of papillary thyroid carcinoma follicular variant according to its histological subtypes (encapsulated vs infiltrative) reveals distinct BRAF and RAS mutation patterns. Mod Pathol 2010;23:1191-200

12. Castro P, Rebocho AP, Soares RJ, et al. PAX8-PPARgamme rearrangement. Is frequently detected in the follicular variant of papillary thyroid carcinoma. $J$ Clin Endocrinol Metab 2006;91:213-20.

13. Goldstein NS, Czako P, Neill JS. Metastatic minimally invasive (encapsulated) follicular and Hurthle cell thyroid carcinoma: a study of 34 patients. Mod Pathol 2000;13:123-30

14. Rosai J. The encapsulated follicular variant of papillary thyroid carcinoma: back to the drawing board. Endocr Pathol 2010;21:7-11.

15. Baloch ZW, LiVolsi VA. Encapsulated follicular variant of papillary thyroid carcinoma with bone metastases. Mod Pathol 2000;13:861-5.

16. Piana S, Frasoldati A, Di Felice E, et al. Encapsulated well-differentiated follicularpatterned thyroid carcinomas do not play a significant role in the fatality rates from thyroid carcinoma. Am J Surg Pathol 2010;34:868-72.

17. Finley DJ, Arora N, Zhu B, et al. Molecular profiling distinguishes papillary carcinoma from benign thyroid nodules. J Clin Endocrinol Metab 2004;89:3214-23. 